\title{
Nonlinear switching with a graphene coupler
}

\author{
Daria A. Smirnova, ${ }^{1}$ Andrey V. Gorbach, ${ }^{2}$ Ivan V. Iorsh, ${ }^{3}$ Ilya V. Shadrivov, ${ }^{1}$ and Yuri S. Kivshar ${ }^{1,3}$ \\ ${ }^{1}$ Nonlinear Physics Center, Research School of Physics and Engineering, Australian National University, Canberra ACT 0200 , Australia \\ ${ }^{2}$ Centre for Photonics and Photonic Materials, Department of Physics, University of Bath, Bath BA2 7AY, United Kingdom \\ ${ }^{3}$ National Research University of Information Technologies, Mechanics and Optics (ITMO), St. Petersburg 197101, Russia
}

(Received 14 May 2013; published 31 July 2013)

\begin{abstract}
We study nonlinear propagation of electromagnetic waves in two closely spaced graphene layers and demonstrate that this double-layer graphene waveguide can operate as an efficient nonlinear optical coupler for both continuous plasmons and for subwavelength spatial optical plasmon solitons. We analyze the nonlinearity-induced effects of light localization and symmetry breaking in such a graphene coupler, and predict that the interlayer power-dependent coupling provides a mechanism for optical beam control and manipulation at realistic input power levels.
\end{abstract}

DOI: 10.1103/PhysRevB.88.045443 PACS number(s): 78.67.Wj, 42.65.Wi, 73.25.+i, 78.68.+m

\section{INTRODUCTION}

Graphene is a two-dimensional crystal of carbon atoms, which exhibits remarkable characteristics. ${ }^{1}$ Recently, its unique optical properties have generated significant interest in the research community (see, e.g., Refs. 2 and 3). An optical response of graphene is characterized by a surface conductivity which is related to graphene's chemical potential and Fermi energy. At certain frequencies, graphene behaves as a metal, and its coupling to electromagnetic waves may support different types of surface plasmon polaritons, which are described theoretically ${ }^{4-6}$ and have been already observed in experiments. ${ }^{7,8}$ These features make graphene a promising material for plasmonics, paving a way towards the development of optical metadevices. ${ }^{9}$

It was shown that a single graphene layer can support both transverse magnetic (TM) and transverse electric (TE) polarized plasmons, ${ }^{10}$ and the wave dispersion properties can be changed by applying an external gate voltage. This is the effect behind recently suggested new types of tunable metamaterial structures based on graphene. ${ }^{11-14}$

Nonlinear optical properties of graphene structures have attracted attention only recently. Large values of nonlinear optical susceptibilities have been predicted theoretically, ${ }^{15,16}$ and recently they were verified experimentally for the thirdorder nonlinear response. ${ }^{17}$ This finding opens a way for the exploration of strong nonlinear photonic effects in graphene structures, including nonlinear self-action of surface plasmons in graphene ${ }^{18}$ and the generation of subwavelength spatial solitons. $^{19}$

In this paper, we study analytically and numerically the nonlinear propagation of light in two coupled layers of graphene, and demonstrate that this simple double-layer structure can operate as an efficient optical coupler for both continuous plasmon polaritons and for subwavelength spatial solitons. We demonstrate the nonlinearity-induced symmetry breaking in this graphene coupler and discuss a physical mechanism for optical beam control and manipulation. We show that in order to achieve nonlinear functionality, graphene should exhibit low loss, and we discuss related implications.

\section{MODEL}

We consider a planar structure created by two parallel layers of graphene, as shown schematically in Fig. 1. We assume that the surrounding dielectric material is a homogeneous medium with the dielectric permittivity $\varepsilon$, and study the nonlinear propagation of plasmons in the layers. To describe the interaction of plasmons excited in each layer of our structure, we generalize the analytical method developed recently in Ref. 18. We start with Maxwell's equations describing the propagation of monochromatic electromagnetic waves with the field dependencies $\sim \exp (-i \omega t)$,

$$
\begin{aligned}
& \nabla \times \mathbf{E}=i k_{0} \mathbf{H}, \\
& \nabla \times \mathbf{H}=-i k_{0} \varepsilon \mathbf{E}+\frac{4 \pi}{c}[\delta(x+d / 2)+\delta(x-d / 2)] \mathbf{J},
\end{aligned}
$$

where $k_{0}=\omega / c$ is the wave number in free space, $\omega$ is the angular frequency, and $c$ is the speed of light. We assume that the graphene layers are placed at $x= \pm d / 2$, as indicated by Dirac's delta functions $\delta$, with $\mathbf{J}$ being the current density induced in the graphene layers.

In the linear regime, the induced current is proportional to the tangential component of the electric field, $\mathbf{J}=\sigma \mathbf{E}_{\tau}$, where $\sigma \equiv \sigma^{(R)}+i \sigma^{(I)}$ is a linear frequency-dependent surface conductivity of graphene. Each isolated graphene layer supports localized surface plasmons with the TM polarization. ${ }^{4,6}$ When losses are neglected, the magnetic field of these plasmon modes has the form

$$
\mathbf{H}_{1,2}^{(0)}=\mathcal{A}_{1,2} h_{1,2}(x) e^{i k_{0} \beta z} \mathbf{y}_{\mathbf{0}},
$$

with the transverse mode profile given by

$$
h_{1,2}(x)=i k_{0}\left(\frac{\varepsilon}{\kappa}\right) e^{-\kappa|x \pm d / 2|} \begin{cases}1, & x>\mp d / 2, \\ -1, & x<\mp d / 2,\end{cases}
$$

where the upper and lower signs correspond to the subscripts 1 and 2 and are associated with the layers located at $x=-d / 2$ and $x=d / 2$, respectively (cf. Fig. 1), $\kappa=k_{0} \sqrt{\beta^{2}-\varepsilon}$, and the normalized wave number $\beta$ is found from the dispersion relation

$$
\frac{2 \varepsilon}{k_{0} \sqrt{\beta^{2}-\varepsilon}}=\frac{4 \pi}{\omega} \sigma^{(I)} \text {. }
$$




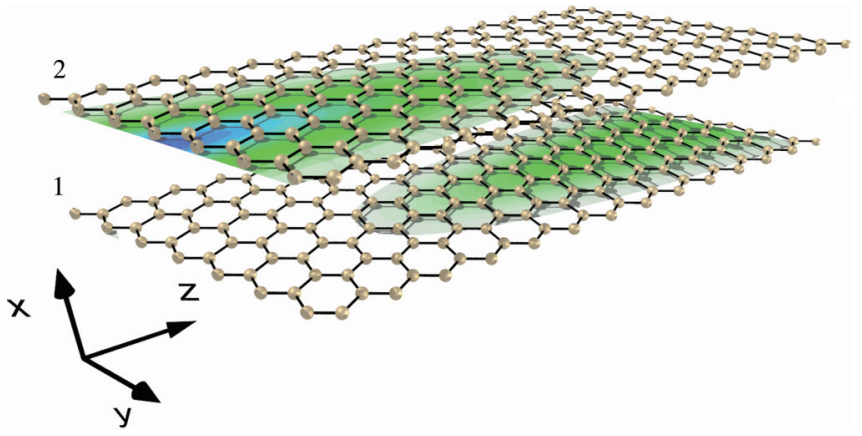

FIG. 1. (Color online) Schematic of a nonlinear graphene coupler composed of two layers of graphene. The color pattern demonstrates how a plasmon beam excited in the top layer tunnels to the bottom layer (numerical results not to scale). The bottom layer (which we call layer 1) and top layer (layer 2) are located in the planes $x=-d / 2$ and $x=d / 2$, respectively.

To describe the propagation of nonlinear plasmons in this double-layer graphene structure, we use the slowly varying envelope approximation usually employed in the physics of optical solitons. ${ }^{20}$ To be more specific, for our problem we study a weakly dissipative case when $\sigma^{(R)} \ll \sigma^{(I)}$. This dissipation is low when the frequency of the plasmons and Fermi energy of graphene satisfy the relations $\hbar \omega<1.67 \mathcal{E}_{F}\left(\sigma^{(I)}>0\right)$, $k_{B} T \ll \mathcal{E}_{F} .{ }^{21}$ We also neglect spatial dispersion, which is valid for $\hbar \omega \leqslant \mathcal{E}_{F}{ }^{21}$

To utilize the asymptotic expansion approach, we assume that (i) nonlinear correction to the conductivity $\sigma$ is small, (ii) waves are localized in the $y$ direction, and their width $\Lambda$ is such that diffraction remains weak, and (iii) evanescent coupling between plasmons excited in different graphene layers is small. Under these assumptions, the induced current can be taken in the form ${ }^{18}$

$$
\begin{aligned}
\mathbf{J} & =\hat{\sigma} \mathbf{E}_{\tau}=\left(\sigma+\sigma^{\mathrm{NL}}\left|E_{\tau}\right|^{2}\right) \mathbf{E}_{\tau}, \\
\sigma^{\mathrm{NL}} & =\frac{\sigma_{3}}{4}\left(3+\frac{\beta^{2}}{\beta^{2}-\varepsilon}\right),
\end{aligned}
$$

where $\sigma_{3}$ is the third-order nonlinear conductivity of graphene. ${ }^{22}$ To develop a consistent perturbation theory, we further assume that in the resulting equations the terms responsible for the above mentioned effects are of the same order of smallness. Now we formally introduce a small parameter $\mu$,

$$
\mu^{2}=\max \left\{\left|\frac{\sigma^{(R)}}{\sigma^{(I)}}\right|,\left|\frac{\sigma^{\mathrm{NL}}\left|E_{\tau}\right|^{2}}{\sigma^{(I)}}\right|,\left(k_{0} \beta \Lambda\right)^{2}, e^{-k_{0} \sqrt{\left(\beta^{2}-\varepsilon\right) d}}\right\},
$$

and derive the equations for slowly varying plasmon amplitudes. We rewrite Maxwell's equations (1) as follows:

$$
\begin{aligned}
& \nabla \times \mathbf{E}_{1,2}=i k_{0} \mathbf{H}_{1,2}, \\
& \nabla \times \mathbf{H}_{1,2}=-i k_{0} \varepsilon \mathbf{E}_{1,2}+\frac{4 \pi}{c} \delta(x \pm d / 2) \hat{\sigma}\left(\mathbf{E}_{1,2 \tau}+\mathbf{E}_{2,1 \tau}\right),
\end{aligned}
$$

so that the superpositions $\mathbf{E}=\mathbf{E}_{1}+\mathbf{E}_{2}$ and $\mathbf{H}=\mathbf{H}_{1}+\mathbf{H}_{2}$ satisfy the original equations. Then, developing a perturbation theory, the solutions of Eqs. (5) are sought in the form of the following asymptotic series:

$$
\begin{aligned}
H_{1,2 y}= & {\left[\mathrm{A}_{1,2}\left(\mu^{2} \mathbf{z}, \mu \mathbf{y}\right) h_{1,2}(x)\right.} \\
& \left.+\mu^{2} \mathbf{H}_{1,2}^{(2)}\left(\mu^{2} \mathbf{z}, \mu \mathbf{y}, x\right)+\cdots\right] e^{i k_{0} \beta z} \\
= & {\left[\mathcal{A}_{1,2}(z, y) h_{1,2}(x)+H_{1,2}^{(2)}(z, y, x)+\cdots\right] e^{i k_{0} \beta z}, } \\
H_{1,2 z}= & {\left[\mu \mathrm{H}_{1,2}^{(1)}\left(\mu^{2} \mathbf{z}, \mu \mathbf{y}, x\right)\right.} \\
& \left.+\mu^{3} \mathrm{H}_{1,2}^{(3)}\left(\mu^{2} \mathbf{z}, \mu \mathbf{y}, x\right)+\cdots\right] e^{i k_{0} \beta z} \\
= & {\left[H_{1,2}^{(1)}(z, y, x)+H_{1,2}^{(3)}(z, y, x)+\cdots\right] e^{i k_{0} \beta z} . }
\end{aligned}
$$

The zero-order term in $\mu$ returns the identity for the unperturbed plasmons (2)-(4). The correction $H_{1,2}^{(1)}$ of the first order in $\mu$ is determined from $\boldsymbol{\nabla} \cdot \mathbf{H}_{1,2}=0$ as

$$
H_{1,2}^{(1)}=\frac{i}{k_{0} \beta} \frac{\partial \mathcal{A}_{1,2}}{\partial y} h(x) .
$$

In the order of $\mu^{2}$ we obtain

$$
\begin{aligned}
\frac{d^{2} H_{1,2}^{(2)}}{d x^{2}} & +k_{0}^{2}\left(\varepsilon-\beta^{2}\right) H_{1,2}^{(2)}-\frac{4 \pi}{c} i \sigma^{(I)} \dot{\delta}(x \pm d / 2) E_{1,2 z}^{(2)}=F_{1,2} \\
F_{1,2}= & -\left(2 i k_{0} \beta \frac{\partial \mathcal{A}_{1,2}}{\partial z}+\frac{\partial^{2} \mathcal{A}_{1,2}}{\partial y^{2}}\right) h_{1,2}(x) \\
& +\frac{4 \pi}{c} \dot{\delta}(x \pm d / 2)\left(\sigma^{(R)}+\sigma^{\mathrm{NL}}\left|\mathcal{A}_{1,2}\right|^{2}\right) \mathcal{A}_{1,2} \\
& +\frac{4 \pi}{c} i \sigma^{(I)} \dot{\delta}(x \pm d / 2) \mathcal{A}_{2,1} e^{-\kappa d}
\end{aligned}
$$

where $\dot{\delta}$ is the derivative of the Dirac's delta function, and the corrections

$$
E_{1,2 z}^{(2)}=\frac{i}{k_{0} \varepsilon} \frac{\partial H_{1,2}^{(2)}}{\partial x}
$$

are continuous at $x= \pm d / 2$.

Then, applying the Fredholm theorem, ${ }^{23}$ which states that the solution for the correction $H_{1,2}^{(2)}$ is nondiverging if the eigenmodes of the homogeneous equation for $H_{1,2}^{(2)}$ are orthogonal to the perturbation, we finally derive the nonlinear equations for the slowly varying envelopes $\mathcal{A}_{1,2}$ of the TMpolarized plasmons propagating in each layer,

$2 i k_{0} \beta\left(\frac{\partial \mathcal{A}_{1,2}}{\partial z}+\gamma \mathcal{A}_{1,2}\right)+\frac{\partial^{2} \mathcal{A}_{1,2}}{\partial y^{2}}+g\left|\mathcal{A}_{1,2}\right|^{2} \mathcal{A}_{1,2}=Q \mathcal{A}_{2,1}$,

where

$$
\begin{aligned}
\gamma & =\frac{2 \pi}{c \varepsilon \beta} \sigma^{(R)}\left(\beta^{2}-\varepsilon\right)^{3 / 2} k_{0}, \\
g & =\frac{4 \pi}{c \varepsilon}\left(\beta^{2}-\varepsilon\right)^{3 / 2} i \sigma^{\mathrm{NL}} k_{0}^{2}, \\
Q & =\frac{4 \pi}{c \varepsilon} e^{-\kappa d} \sigma^{(I)}\left(\beta^{2}-\varepsilon\right)^{3 / 2} k_{0}^{2},
\end{aligned}
$$

are a linear absorption parameter, and nonlinear and coupling coefficients, respectively. Remarkably, Eqs. (9), along with the correct analytical expressions for the effective coefficients (10), can be obtained in the order $O\left(\mu^{2}\right)$ of the perturbation 
expansion by using the substitution

$$
k_{0} \beta=k_{0} \beta+\left(-i \frac{\partial}{\partial z}-\frac{1}{2 k_{0} \beta} \frac{\partial^{2}}{\partial y^{2}}\right)+\cdots
$$

in the modified dispersion relation (4), which in the presence of the second layer takes the form

$$
\begin{aligned}
\frac{2 \varepsilon}{k_{0} \sqrt{\beta^{2}-\varepsilon}}= & \frac{4 \pi}{\omega}\left(\sigma^{(I)}-i\left[\sigma^{(R)}+\sigma^{\mathrm{NL}}\left|\mathcal{A}_{1}\right|^{2}\right.\right. \\
& \left.\left.+i \sigma^{(I)} e^{-\kappa d} \frac{\mathcal{A}_{2}}{\mathcal{A}_{1}}\right]+\cdots\right) .
\end{aligned}
$$

\section{NONLINEAR SWITCHING}

In the framework of the nonlinear amplitude equations (9) disregarding losses and beam diffraction, we can analyze different types of TM-polarized eigenmodes of a nonlinear double-layer graphene waveguide. For further calculations, we employ the following expressions for the graphene conductivity: ${ }^{10,18,22}$

$$
\begin{aligned}
\sigma & =\frac{i e^{2}}{\pi \hbar}\left[\frac{1}{\Omega+i v_{\text {intra }}}+\frac{1}{4} \ln \frac{2-\Omega}{2+\Omega}\right], \\
\sigma_{3} & =-i \frac{3}{32} \frac{e^{2}}{\pi \hbar} \frac{\left(e V_{F}\right)^{2} \hbar^{2}}{\mathcal{E}_{F}^{4} \Omega^{3}},
\end{aligned}
$$

where $\Omega=\hbar \omega / \mathcal{E}_{F}, v_{\text {intra }}=\hbar /\left(\mathcal{E}_{F} \tau_{\text {intra }}\right)$, for the doping level of $\mathcal{E}_{F}=0.1 \mathrm{eV}, \Omega=1\left(\lambda=2 \pi / k_{0} \approx 12.4 \mu \mathrm{m}\right), \tau_{\text {intra }}=10 \mathrm{ps},{ }^{24}$ the Fermi velocity in graphene $V_{F} \approx c / 300, \varepsilon=4$, and take the separation between the layers as $d=28 \mathrm{~nm}$. We take quite a large relaxation time and discuss the implications of this choice below.

We find that three types of nonlinear modes can propagate in this double-layer graphene coupler, namely, a symmetric (S) mode with $\mathcal{A}_{1}=\mathcal{A}_{2}$, an antisymmetric (AS) mode with $\mathcal{A}_{1}=$ $-\mathcal{A}_{2}$, and an asymmetric (A) mode with $\mathcal{A}_{1} \neq \mathcal{A}_{2}$, similar to the case of a nonlinear dimer. ${ }^{25}$ These modes are presented in Fig. 2 through their (a), (b) transverse profiles and (c) power-dependent shift of the nonlinear propagation constant. In the linear regime, only symmetric (S) and antisymmetric (AS) modes exist. However, above a critical power level, a symmetry breaking occurs in this nonlinear system when a different, asymmetric branch (A) emerges, and it describes the nonlinear states where the power is not distributed equally between the lower and upper graphene layers. The asymmetric mode bifurcates at the point " $p$ " from the antisymmetric branch, and it is stable, being characterized by a predominant energy concentration in the vicinity of one of the layers. The AS mode becomes unstable above the critical power.

Next, we focus our attention on the power-controlled switching of plasmon beams between the layers and study numerically the propagation of a sech-like input beam launched into the upper layer (as shown in Fig. 1). The input beam is selected to be the fundamental solution of a single (uncoupled) nonlinear equation, and it describes a spatial soliton. ${ }^{19}$ For a peak power density of $6 \mathrm{~W} / \mathrm{m}$, the soliton beam width is about $50 \mathrm{~nm}$. This significant subwavelength localization is supported by the large Kerr nonlinearity of graphene. ${ }^{18,19}$
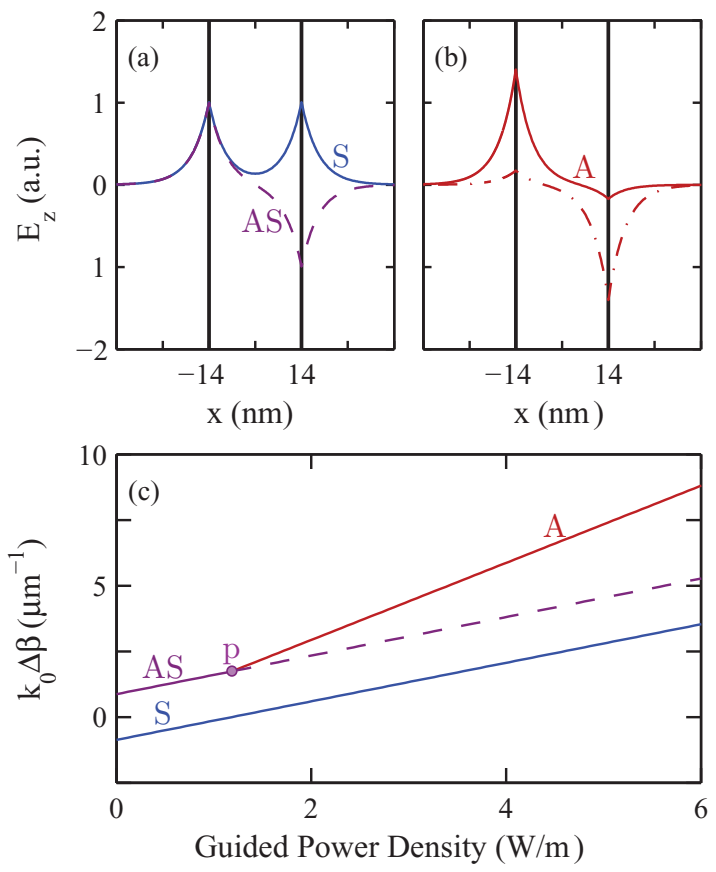

FIG. 2. (Color online) (a), (b) Examples of the transverse mode profiles for symmetric (S), antisymmetric (AS), and asymmetric (A) modes propagating in the double-layer graphene shown for the $E_{z}$ component. (c) Shift of the nonlinear propagation constant vs the mode power density in the guided plasmon mode propagating in the $z$ direction. Solid and dashed lines correspond to stable and unstable branches, respectively.

In Fig. 3, we compare the corresponding switching characteristics of the nonlinear graphene coupler for the continuous plasmons (whose amplitude is constant along y) (dashed curve) and for the beams of a finite extent including the soliton switching (solid curves). The coupler length is selected at the half-beat length $L=\pi /(2 Q)$, when in the linear regime the input power transfers completely into the second layer. ${ }^{20}$ Apart from an increase of the threshold power density, two regimes of the coupler operation can be clearly distinguished.

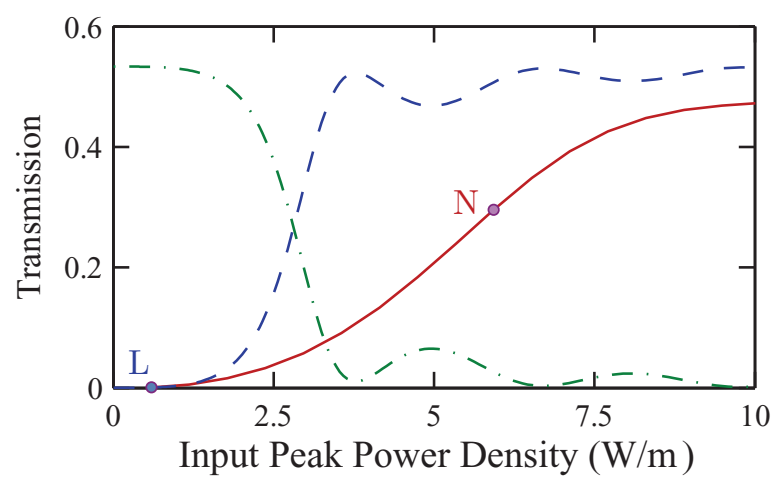

FIG. 3. (Color online) Switching characteristics of the nonlinear graphene coupler. Shown is a fraction of the power transmitted in the pumped layer for a cw (dashed curve) and soliton input beam (solid curve) in a half-beat-length coupler as a function of the input peak power density. Dashed-dotted curve: Calculated relative fractional output power emerging from the other layer in the continuous plasmon regime. 

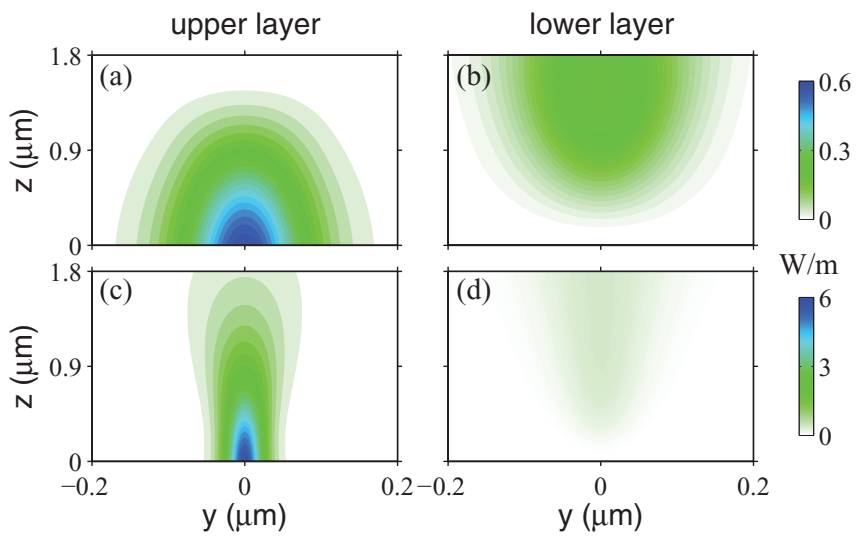

$\mathrm{W} / \mathrm{m}$

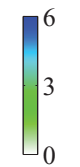

FIG. 4. (Color online) Spatial distribution of the power density in the layers of the nonlinear graphene coupler for different input peak power densities: (a), (b) Linear regime, $P_{\text {in }}=0.6 \mathrm{~W} / \mathrm{m}$ and (c), (d) nonlinear regime, $P_{\text {in }}=6 \mathrm{~W} / \mathrm{m}$, corresponding to the points marked in Fig. 3 as L and N, respectively.

For relatively small input powers, in the so-called linear regime, the plasmon beam of the upper layer couples to the lower layer and tunnels almost completely [see Figs. 4(a) and 4(b)]. For higher values of the input power, the energy transfer between the layers becomes inhibited, and we observe the nonlinear regime when the plasmon beam remains in the upper layer [see Figs. 4(c) and 4(d)]. Importantly, the dissipation does not inhibit switching but it modifies the slope of the curves and the asymptotic value of the switching curve not achieving unity, as shown in Fig. 3. Therefore, the beam may be effectively routed between the graphene layers by changing its input power.

From the power flow $(6 \mathrm{~W} / \mathrm{m})$ and knowing the plasmon structure, we estimate the maximum amplitude of the electric field on graphene layer at $7 \times 10^{6} \mathrm{~V} / \mathrm{m}$, which is below the graphene breakdown threshold. ${ }^{26}$ In our simulations, however, we assumed low loss graphene, with a relaxation time of 10 ps. Such relaxation times were observed in several works with multilayer structures, ${ }^{24,27}$ however, typical single-layer graphene reported so far experimentally usually exhibits relaxation times of the order of $0.1 \mathrm{ps}$. Such graphene with higher losses will have a shorter plasmon propagation length, and it will require larger, and probably unachievable, light intensities. Therefore, we suggest that high quality, low loss graphene will potentially be suitable for creating nonlinear couplers.

Further, we discuss the case when two in-phase beams of an identical shape are launched into both layers, being shifted

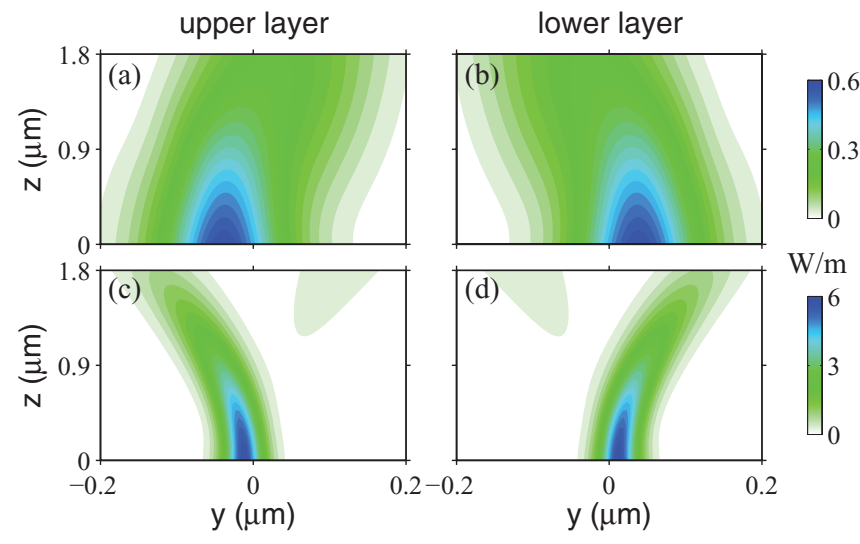

FIG. 5. (Color online) Plasmon beam routing in the nonlinear graphene coupler. Spatial distribution of the power density in the layers of the nonlinear graphene coupler for different input peak power densities: (a), (b) Linear regime, $P_{\text {in }}=0.6 \mathrm{~W} / \mathrm{m}$ and (c), (d) nonlinear regime, $P_{\text {in }}=6 \mathrm{~W} / \mathrm{m}$.

initially by a half of their width with respect to each other. In the linear regime, the power exchange between the layers leads to the effective attraction between the centers of mass of the beams, as can be seen in Figs. 5(a) and 5(b). However, in the strongly nonlinear regime the energy exchange is suppressed and the mutual interaction of optical solitons generated in the two different layers is accompanied by their repulsion, as shown in Figs. 5(c) and 5(d).

\section{CONCLUSIONS}

We have studied analytically and numerically the propagation of nonlinear electromagnetic waves in a double-layer graphene structure. We have revealed that this structure can operate as an efficient planar nonlinear coupler, demonstrating the switching of light between two different layers of graphene. We have studied the nonlinear effects in this graphene coupler for both continuous plasmons and subwavelength spatial solitons, and described the symmetry breaking and nonlinear switching with various opportunities for optical beam control and manipulation at the nanoscale.

\section{ACKNOWLEDGMENTS}

The work was supported by the Australian Research Council, the Engineering and Physical Research Council of the UK under EP/K009397/1, and the Ministry of Education and Science of the Russian Federation. The authors thank Y. Bludov and P. Belov for useful discussions.

\footnotetext{
${ }^{1}$ A. K. Geim, Science 324, 1530 (2009).

${ }^{2}$ R. R. Nair, P. Blake, A. N. Grigorenko, K. S. Novoselov, T. J. Booth, T. Stauber, N. M. R. Peres, and A. K. Geim, Science 320, 1308 (2008).

${ }^{3}$ F. Bonaccorso, Z. Sun, T. Hasan, and A. C. Ferrari, Nat. Photonics 4, 611 (2010).

${ }^{4}$ F. H. L. Koppens, D. E. Chang, and F. J. G. de Abajo, Nano Lett. 11, 3370 (2011).
}

${ }^{5}$ M. Jablan, H. Buljan, and M. Soljacic, Opt. Express 19, 11236 (2011).

${ }^{6}$ A. Y. Nikitin, F. Guinea, F. J. Garcia-Vidal, and L. Martin-Moreno, Phys. Rev. B 84, 195446 (2011).

${ }^{7}$ Z. Fei, A. S. Rodin, G. O. Andreev, W. Bao, A. S. McLeod, M. Wagner, L. M. Zhang, Z. Zhao, M. Thiemens, G. Dominguez, M. M. Fogler, A. H. Castro Neto, C. N. Lau, F. Keilmann, and D. N. Basov, Nature (London) 487, 82 (2012). 
${ }^{8}$ J. Chen, M. Badioli, P. Alonso-Gonzalez, S. Thongrattanasiri, F. Huth, J. Osmond, M. Spasenovic, A. Centeno, A. Pesquera, P. Godignon, A. Z. Elorza, N. Camara, F. J. García de Abajo, R. Hillenbrand, and F. H. L. Koppens, Nature (London) 487, 77 (2012).

${ }^{9}$ N. I. Zheludev and Yu. S. Kivshar, Nat. Mater. 11, 917 (2012).

${ }^{10}$ S. A. Mikhailov and K. Ziegler, Phys. Rev. Lett. 99, 016803 (2007).

${ }^{11}$ A. Vakil and N. Engheta, Science 332, 1291 (2011).

${ }^{12}$ L. Ju, B. Geng, J. Horng, C. Girit, M. Martin, Z. Hao, H. A. Bechtel, X. Liang, A. Zett, Y. R. Shen, and F. Wang, Nat. Nanotechnol. 6, 630 (2011).

${ }^{13}$ S. H. Lee, M. Choi, T. Kim, S. Lee, M. Liu, X. Yin, H. K. Choi, S. S. Lee, C. G. Choi, S. Choi, X. Zhang, and B. Min, Nat. Mater. 11, 936 (2012).

${ }^{14}$ I. V. Iorsh, I. S. Mukhin, I. V. Shadrivov, P. A. Belov, and Yu. S. Kivshar, Phys. Rev. B 87, 075416 (2013).

${ }^{15}$ S. A. Mikhailov, Europhys. Lett. 79, 27002 (2007).

${ }^{16}$ K. L. Ishikawa, Phys. Rev. B 82, 201402 (2010).

${ }^{17}$ E. Hendry, P. J. Hale, J. Moger, A. K. Savchenko, and S. A. Mikhailov, Phys. Rev. Lett. 105, 097401 (2010).
${ }^{18}$ A. V. Gorbach, Phys. Rev. A 87, 013830 (2013).

${ }^{19}$ M. L. Nesterov, J. Bravo-Abad, A. Yu. Nikitin, F. J. Garcia-Vidal, and L. Martin-Moreno, Laser Photonics Rev. 7, L7 (2013).

${ }^{20}$ Yu. S. Kivshar and G. A. Agrawal, Optical Solitons: From Fibers to Photonic Crystals (Academic, San Diego, 2003).

${ }^{21}$ L. A. Falkovsky and A. A. Varlamov, Eur. Phys. J. B 56, 281 (2007).

${ }^{22}$ S. A. Mikhailov and K. Ziegler, J. Phys.: Condens. Matter 20, 384204 (2008).

${ }^{23}$ G. A. Korn and T. M. Korn, Mathematical Handbook for Scientists and Engineers (McGraw-Hill, New York, 1961).

${ }^{24}$ T. Otsuji, S. A. Boubanga Tombet, A. Satou, H. Fukidome, M. Suemitsu, E. Sano, V. Popov, M. Ryzhii, and V. Ryzhii, J. Phys. D: Appl. Phys. 45, 303001 (2012).

${ }^{25}$ J. C. Eilbeck, P. S. Lomdahl, and A. C. Scott, Physica D 16, 318 (1985).

${ }^{26}$ M. Currie, J. D. Caldwell, F. J. Bezares, J. Robinson, T. Anderson, H. Chun, and M. Tadjerb, Appl. Phys. Lett. 99, 211909 (2011).

${ }^{27}$ P. Neugebauer, M. Orlita, C. Faugeras, A.-L. Barra, and M. Potemski, Phys. Rev. Lett. 103, 136403 (2009). 